\title{
THE NECESSITY FOR DEVELOPING SKILL AT Mind-PROCESS OBSERVATION, AND A Suggested Methodology
}

\author{
Copthorne Macdonald* \\ Charlottetown, Prince Edward Island, \\ C1A 8C5 Canada \\ Received September 7, 2010; accepted September 14, 2010
}

\begin{abstract}
Ever since introspectionism was abandoned early in this century, the scientific community has shown little interest in training people to observe the functioning of their own minds with greater than ordinary clarity. Thus, despite advanced academic training and much knowledge about physical phenomena and processes, few consciousness theorists or experimentalists have any special expertise at closely observing mental artifacts and processes. Without observational clarity about the mental, how is that web of physical/mental correlations going to be mapped?
\end{abstract}

Key words: Attention; Consciousness; Mind; Observation

\section{INTRODUCTION}

Unfortunately, we humans are not naturally skilled at observing mind content and process. Our natural tendency is to get lost in the mental drama that accompanies our life. We tend to identify with the informational content of our minds in much the same way that we identify with the informational content of an engrossing movie. Consequently, much of the time we are "lost in the show." We have developed neither the detachment required for scientifically sound observation of mind contents and functions, nor the requisite observational skills.

\section{THE NEED FOR ACCURATE MENTAL DATA}

Increasingly, situations arise where knowledge from one discipline alone is not enough to extend the frontiers of understanding and creative capability. In order to do creative work in the field of medical technology, for example, the biomedical engineer must have both physician-type knowledge and engineer-type knowledge in one brain/mind. I am suggesting that a similar necessity exists in the field of consciousness studies. Whether their primary discipline is philosophy, neurology, psychology, or cognitive science, those theoreticians and researchers who complement academically grounded expertise with highly developed mind-watching skills have an advantage. They bring two pools of relevant data to their explanation-creating, theory-building activities. This, I suggest, gives them a significant advantage in their efforts to arrive at accurate, insightful explanations of what is going on.

\section{MIND-WATCHING METHODOLOGIES}

\section{Introspectionism's legacy}

Experimental introspectionism was the psychologist's tool of choice in the late 19th Century. The experiments themselves tended to be limited in scope. Each was designed to explore some limited aspect of subjective experience, and the inner observation was often correlated with some objective factor such as time or the intensity of a sensation. In his Principles of Psychology, William James (1950, p. 191) acknowledged that "introspection is difficult and fallible," but went on to say that "the difficulty is simply that of all observation of whatever kind." Titchener (1908, p.180) put it this way: "There is no difference, in principle, between inspection and introspection."

It is interesting to review the mindset and terminology of the Introspectionists - the kinds of distinctions they 
made in their observations. Titchener (1908), for example, spoke of the quality, intensity, temporal duration, spatial extension, and attentional clearness of sensations. He also acknowledged the necessity for experience in mind watching if anything worthwhile was to come of it, and gave this example (1908):

"When a tachistoscopic field is exposed for the first time to an unpractised observer, he will very probably fail to 'make out' anything at all; the lines or letters or geometric figures are seen as a general impression, without the discrimination of detail." (p. 238)

It is this "discrimination of detail" that observational practice helps develop, and which must be present if examination of mind contents is to have significant value. Titchener (180, p. 179) speaks of the "practised observer" having "the introspective habit . . . ingrained in the texture of his mind," making it possible "to take mental notes while the observation is in progress" and even, at times, to "jot down written notes as the histologist does while his eye is still held to the ocular of the microscope."

\section{The present necessity}

The experimental introspectionism of Wundt, Külpe, and Titchener involved, for the most part, a focused one-thing-at-a-time kind of looking. It was appropriate to the psychological questions of that day, and the data it provided fed the inductive processes of those experimenters.

Today we have a different set of problems. A major concern of experimenters and theorists these days is the relationship of brain process to mind process - the relationship of neural activity to mental qualia and functioning. I am suggesting that a slightly different kind of introspective looking can contribute to the development of this kind of understanding. Many years ago Boring spoke of Köhler's plea for "phenomenology as free description of, without analysis into formal elements" (Boring, 1950, p. 601), and quoted Köhler as saying "Never, I believe, shall we be able to solve any problems of ultimate principle . . until we use the phenomenological method, the qualitative analysis of experience." Today, I'm simply echoing Köhler's plea.

Specifically, I am suggesting that mind content and function be watched with the same sort of observational skill that naturalists employ when they observe the behavior of wildlife: a combination of detachment, wide attentional focus, keen interest, alertness, and the ability to detect fast-changing subtlety.

This is a less directed kind of mind watching than that practiced by the turn-of-the-century introspectionists. One watches, not focused on one specific thing, but without expectation, simply waiting to see what happens next. One watches to see what arises, and to detect (if possible) causal patterns related to those arisings.
The bad news is that this kind of observational capability is not innate. Naive observers just don't have it. The good news is that this combination of attitudes and skills can be developed through practice. The experienced naturalist on a field trip sees much more than she did on field trips when she was a student. The bird watcher with 20 years experience identifies many more birds than does the beginner. Similarly, the ability to see what is happening in the mind with relative clarity and precision develops through mind-watching practice.

The sort of mental training required is already going on in many parts of the world, though not for this specific purpose. It is found in the monastic disciplines of Buddhism, and in Western methodologies such as Open Focus (Fehmi and Fritz, 1980).[2] In Theravadin Buddhism the relevant technique is variously called Vipassana, Mindfulness, or Insight meditation.[3] In Zen, the terms used are Bare Attention, and ShikanTaza.[4] In Tibetan Buddhism, it is Dzogchen practice[5] that helps develop this observational skill.

In all these practices, the methodology is to watch the ever-changing qualia and dynamic processes of mind with an interested, caring detachment. Skill at doing this develops over time, and effective practice involves engaging in this kind of mind watching daily - for perhaps an hour - and in the Buddhist variations on this theme, periodically for days- or weeks-at-a-time in intensive retreat situations.

\section{The Buddhist approach}

In the Buddhist practices, the observer first develops attentional steadiness or concentration by spending time watching just one thing. This "one thing" is usually the sensations that arise in connection with breathing either the sensation of the breath passing through the nostrils, or sensations connected with the rising and falling of the abdomen. Because the body is always breathing, and because these breath-associated sensations are relatively subtle, they make good objects of attention. When the ability to watch breath sensations has developed to a certain point, the observer is encouraged to widen the focus of attention to include other objects - physical sensations, feelings, sounds, thoughts - and ultimately, whatever arises in the mind. Theravadin Buddhism's Vipassana, Zen Buddhism's Shikan-Taza and Tibetan Buddhism's Dzogchen all involve practicing this non-identified, accepting, open-toeverything kind of noticing.

\section{Open Focus}

The Open Focus approach skips the narrow-focus, onepointed initial practice of the Buddhist traditions, and instead attempts to talk the participant directly into the second, "open" kind of mind watching through the use of pre-recorded audio tapes and CDs. 


\section{TANGIBLE RESULTS}

When you follow this methodology to the point that you start to get results, what is it that you are able to see clearly that others tend to miss? The following examples illustrate a few of the possibilities.

1. The automaticity of mind processes, their mechanical nature.

Often noticed during the very first attempt at this sort of mind watching is the lack of personal control over mind happenings. The teacher's instruction is simple: "Sit for the next 45 minutes and pay attention to the physical sensations that accompany breathing." Doing this on the first attempt proves impossible. At some point - perhaps as early as one minute into the process - attention gets diverted from breath sensations to thoughts, or sounds, or an itch. As Daniel Goleman (1988) describes it

"At the outset, the meditator's focus wanders from the object of meditation. As he notices he has wandered, he returns his awareness to the proper focus. His onepointedness is occasional, coming in fits and starts. His mind oscillates between the object of meditation and distracting thoughts, feelings, and sensations." (p. 10)

Later, concentration develops and attention is able to remain on the chosen object. Still, even though attention is now stable, other mind content continues to arise. Goleman continues:

"The first landmark in concentration comes when the meditator's mind is unaffected both by outer distractions, such as nearby sounds, and by the turbulence of his own assorted thoughts and feelings. Although sounds are heard, and his thoughts and feelings are noticed, they do not disturb the meditator." (p. 10)

("Disturb" in this context means causing attention to move away from the chosen object.)

2. The relationship between awareness and the informational content of mind.

If the mind watching effort is pursued for an extended period, it eventually becomes apparent that even when the mind is very "quiet" (little mind content: quiet surroundings, closed eyes, few thoughts arising), one is still intensely aware. As I once put it (Macdonald, 1993):

"A moment may come... when awareness becomes aware of awareness - when the observing faculty becomes aware of itself as an entity separate in some sense from the show, and different in nature. At such moments it becomes clear that awareness is inherently still and unchanging, and that all motion, all change, resides in the informational show...." (p. 89)

What sense a person makes of this observation may be influenced by the kinds of knowledge that the person brings to it. I happened to bring an understanding of communication systems engineering to the experience, and the mental data I observed seemed to fit a particular conceptual model from that field.
In radio transmission, an energy carrier is modulated by program information, by the show. The carrier is, in effect, a medium which has the informational message impressed upon it, and which then carries that message with it. My mind watching led me to see awareness as a medium, as a kind of sentient carrier. This awareness carrier was being modulated by brain-generated information, and the products of that modulation process were mental artifacts: mind content, qualia. I came to see qualia as instances of information-modulated awareness. Going into this a bit further (Macdonald, 1993):

"At times I speak of awareness and the informational show as being separate - and from one perspective they are. But from another perspective they are not. ... [In the radio example] the show modulates the carrier (changing the carrier's frequency in FM, or its amplitude in AM). The physical reality is the carrier itself; the show is a changing informational pattern encoded in the instant-to-instant changes impressed on the carrier. The show and the carrier are separate conceptually; they are different kinds of reality. At the same time, they are one in the physical reality of an energy carrier undergoing modulation. Similarly, awareness and the mental show are conceptually different kinds of things, and in that sense are separate. They are one, however, in the reality of awareness undergoing modulation." (p. 90)

Using another analogy, waves are informational modulations of ocean. From one perspective waves are informational entities in their own right, having distinct shapes and locations. From another perspective, however, waves are simply ocean.

This carrier/information view led to additional writing on the nature of mental and physical reality (Macdonald, 1994) and (Macdonald, 2010), as I explained (Edgar, 1995):

My interest in new descriptions of reality arose out of my need to make sense of two widely different poles of personal experience. As an electronic design engineer, I had developed confidence in the validity of science and the intellect. Science works. By following scientific laws I had been able to design sophisticated physical systems that never before existed. Then I discovered meditation, a quiet mind, and the deep intuitive side of myself. In the process of spending several thousand hours intently watching mental happenings, certain truths about subjective experience became equally clear, equally persuasive. I found myself with one foot in each of two very different worlds. I reasoned that if both worlds were grounded in reality then it should be possible to find (or create) a paradigm capable of encompassing both. Eventually, key pieces (some from communications engineering) fell into place, and the result was the medium/message or carrier/information interpretation of reality presented in Toward Wisdom and the Zygon paper. (p. 258) 


\section{INSIGHTS INTO THE IDENTIFICATION PROCESS}

3 . For many observers the kind of looking we are considering here sheds light on identity and the identification process. One typical insight involves seeing (as in 1. above) that much of what goes on in the mind happens automatically without any sense of intention or personal ownership associated with it. Another (much rarer) identity-related event is a gestalt shift of identification from the body and mind contents to awareness. The "I sense" becomes associated with awareness itself. Such a shift is actively sought in traditions such as Vedanta, Taoism, and some branches of Buddhism. The traditional "Perennial Philosophy" way of viewing the situation was articulated by Aldous Huxley (1945):

"The ground in which the multifarious and time-bound psyche is rooted is a simple timeless awareness. By making ourselves pure in heart and poor in spirit we can discover and be identified with this awareness." (p. 29) Whether or not this particular identity shift is directly experienced, alert but detached mind watching can shed light on our identifications and on the processes that underlie them.

4. An appreciation of other, non-intellectual, modes of knowing.

Our late-20th-century minds tend to be noisy minds, full of informational content and fast-paced mental activity. Because it is extremely difficult (perhaps impossible) to pay close attention to something and engage in discursive thinking at the same time, the kind of attentive mind watching we're talking about tends to quiet the mind. This quieting of the mind has been observed by myself and others to lead to a closer connection with, and appreciation for, the source of guidance and creative solutions often termed intuition, or the intuitive process (Bastick, 1982, p. 277; Goldberg, 1983, p. 17980; Macdonald, 1993).

The phenomenon of knowing it by being it - knowledge through identification - is another sometime byproduct of the quiet mind.

5 . Insights into the role of selective attention.

As Eccles has pointed out, it seems highly unlikely that sophisticated human-level conscious processes would have evolved if they did not have survival and/or reproductive value for the species (Eccles \& Robinson, 1985, p. 37). In other words, consciousness must be effective and play one or more valuable roles. What might that role or roles be?

Mind-watching, coupled with some reading on the subject, led me to conclude that consciousness and the mechanism of selective attention keep the amount of neural computation needed for situation evaluation down to a level that can be handled by a human brain of the present size. As I recently put it (Macdonald, 2010):

"Selective attention appears to be the mechanism that allowed mind to play this operational [situation evalua- tion] role. Theorist of evolution Jerison (1973, p. 4) said: 'I regard the mind and conscious experience as constructions of nervous systems to handle the overwhelming amount of information that they process.' And selective attention appears to be the mechanism that allowed the amount of computation needed for situation evaluation to be kept at a manageable level.

Evolution's mental-physical approach seems to have been this: Create neuronal systems that generate mental metaphors or analogs of the immediate physical situation, and by superposing them bring them together in one mental 'space.' Combine with this a selective attention mechanism which allows the superposed mental fields to be scanned for qualia having survival or reproductive significance. And when attention dwells on a particular quale, arrange for the neuronal correlates of that quale to become available for unconscious computational processing.

If this processing deems the quale significant, my own personal experience indicates that it initiates one of several possible responses:

1. a direct behavior (as when someone jumps immediately to save a child in danger),

2. a subjective 'message' such as a thought, feeling, or emotion which then appears in the mind along with the information already present there, or

3 . an instruction to the systems which control attention to stop paying attention to the present object and resume attentional scanning."

6. Insights into how reactive emotion works.

As I say elsewhere (Macdonald, 1995):

"Strong emotions were another part of evolution's solution to this decision-making and behaviour-guiding problem. In a frog, the automatic reaction to a perceived opportunity or danger is the action itself: go after the fly, jump off the lily pad. In a human being the automatic reaction to an opportunity or danger is likely to be an emotion rather than a direct action. Emotions are messages that suggest or promote certain kinds of action, but they don't initiate action directly. Instead, emotions appear on the screen of mind along with perceptions, thoughts, and other forms of mental data. The message that an emotion presents is taken into account by the situation-evaluation and decision-making process, but it is just one of many pieces of data being considered." (p. 68)

At another point I became aware of the process by which highly-charged emotional states are created. In short, brief impulses of reactive emotion lead to discursive thinking or storymaking which leads to more impulses and more storymaking in positive feedback fashion. If the cyclical feedback continues, that first isolated emotional impulse becomes a full-blown emotional state. (Macdonald, 1993):

"Once having noticed an impulse there are three ways of dealing with it — not all of them equally appropriate: 
1. We can prolong the impulse by identifying with it and weaving a story around it, by feeding energy into a process that maintains it. If we do this, then a state of anger, fear, jealousy, or desire arises. This, in turn, may result in anger-based, fear-based, jealousy-based, or desire-based action of some sort.

2. Another option is to deny or repress the impulse - to push it into the subconscious. This is apt to have unfortunate consequences later, since repressed material is not really gone. It often returns and causes trouble.

3. A third option is simply to notice the impulse, realize its automatic, mechanical, ancient-brain origin, and let it go." (p. 61)

\section{PITFALLS AND LIMITATIONS}

Introspection has its pitfalls and limitations. If we choose to go this route and add mind watching to our bag of tricks, we must recognize the dangers, and not expect more from the methodology than it can deliver. One pitfall is just plain getting it wrong. James (1950) said:

"Something is before us; we do our best to tell what it is, but in spite of our good will we may go astray and give a description more applicable to some other sort of thing. The only safeguard is in the final consensus of our farther knowledge about the thing in question, later views correcting earlier ones, until at last the harmony of a consistent system is reached. Such a system, gradually worked out, is the best guarantee the psychologist can give for the soundness of any particular psychologic observation which he may report." (pp. 191-92)

Memory is an integral part of the process, and memory's limitations must be acknowledged and circumvented to the extent possible. Recording observations immediately, while they still reside in short-term memory, has clarity and accuracy advantages - but interrupts the flow of observation. Waiting till later to take notes avoids the interruption, but risks the erosion of clarity/accuracy as memory degrades. Fortunately, many situations and types of mind content arise over and over again. This allows us to take one approach this time, and another approach the next time, reducing the importance of any one observation.

Yet another potential pitfall is the influence of observation itself on what is observed. This effect varies from insignificant, or practically so, to highly significant. The kind of interruption mentioned above is one manifestation of it. As Titchener (1908, p. 177) put it, "if you try to report the changes in consciousness while these changes are in progress, you interfere with consciousness; your translation of the mental processes into words introduces new factors into the experience itself." Even more intrusive is the effect of observation on emotions. Titchener (1908, p. 177) acknowledged that "Cool consideration of an emotion is fatal to its very existence; your anger disappears, your disappointment eva- porates, as you examine it." (This phenomenon is, of course, sought after and welcomed in the usual use of this methodology: spiritual development.)

\section{CONCLUSION}

The kind of introspection suggested here is no panacea for the ills that beset the field of consciousness studies, but is, I believe, an activity that can facilitate progress. Although this kind of mind observation yields little quantitative information, it does yield qualitative insights and instances of intuitive clarity. Some of these, coupled with objective data, can be crafted by the intellect into testable hypotheses and helpful theoretical constructs. Phenomenological observation, by itself, is not the answer to anything. But it allows a kind of crossfertilization with informational and conceptual content from neurology, psychology, philosophy, and cognitive science that promises to lead to new ways of looking at brain/mind issues, and ultimately to new explanations of, and clarity about, the brain/mind reality.

\section{NOTES}

[1] P.O. Box 2941, Charlottetown, Prince Edward Island, Email: cop@copmacdonald.com

Web sites:

http://www.copmacdonald.com/, http://www.wisdompage.com/, and http://mattersofconsequence.com/

[2] Princeton Biofeedback Center, P.O. Box 572, Princeton, NJ 08540. 609-924-0785.

[3] See (Levine, 1979; Goldstein, 1983; Macdonald, 1993). Also, Insight Meditation Society, Pleasant Street, Barre, MA 01005. 508-355-4378.

[4] See (Kapleau, 1965).

[5] See (Reynolds, 1989; Dowman, 1994; Norbu, 1989).

\section{REFERENCES}

Bastick, T. (1982). Intuition: How We Think and Act. Chichester: John Wiley \& Sons.

Dowman, K. (1994). The Flight of the Garuda: Teachings of the Dzokchen Tradition of Tibetan Buddhism. Boston: Wisdom Publications.

Eccles, J. \& Robinson, D.N. (1985). The Wonder of Being Human: Our Mind and Our Brain. Boston: New Science Library.

Edgar, K.J. (Ed.) (1995). Contemporary Authors. Vol. 145, Detroit: Gale Research.

Fehmi, L.G. \& Fritz, G. (1980). Open Focus: The Attentional Foundation of Health and Well-Being. SOMATICS, Spring, 1980.

Goldberg, P. (1983). The Intuitive Edge: Understanding Intuition and Applying It in Everyday Life. Los Angeles: Jeremy P. Tarcher.

Goldstein, J. (1983). The Experience of Insight: A Simple and Direct Guide to Buddhist Meditation. Boston: Shambhala Publications.

Goleman, D. (1988). The Meditative Mind: The Varieties of Meditative Experience. Los Angeles: J. P. Tarcher. 
Huxley, A. (1945). The Perennial Philosophy. New York: Harper and Brothers.

James, W. (1950). The Principles of Psychology, Vol. 1. New York: Dover Publications.

Jerison, H. J. (1973). Evolution of the Brain and Intelligence. New York: Academic Press.

Kapleau, P. (1967). The Three Pillars of Zen. Boston: Beacon Press.

Levine, S. (1979). A Gradual Awakening. New York: Anchor Books.

Macdonald, C. (1993). Toward Wisdom: Finding Our Way to Inner Peace, Love and Happiness. Toronto: Hounslow Press.

Macdonald, C. (1994). An Energy/ Awareness/ Information Interpretation of Physical and Mental Reality. Zygon: Journal of Religion and Science, 29, 135-51.

Macdonald, C. (1995). Getting a Life: Strategies for Joyful and Effective Living. Toronto: Hounslow Press.

Macdonald, C. (2010). Implications of a Fundamental Consciousness. Activitas Nervosa Superior, 52, 85-93.

Norbu, N. (1989), Dzogchen the Self-Perfected State (London: Penguin/Arkana).

Reynolds, J.M. (1989). Self-Liberation through seeing with naked awareness. Barrytown, NY: Station Hill Press. 